\title{
Hepatic hydrothorax
}

\author{
Nauroz Syed ${ }^{1}$ and Matthew D. Alvin ${ }^{* 2}$ \\ ${ }^{1}$ University of Maryland Medical Center, Baltimore, USA \\ ${ }^{2}$ The Johns Hopkins Hospital, Baltimore, USA
}

\begin{abstract}
Hepatic hydrothorax is a relatively uncommon complication of cirrhosis, which generally portends a poor prognosis. The pathogenesis, evaluation, and management of hepatic hydrothorax is herein reviewed, based on current literature. In general, treatment focuses on symptomatic relief and prevention of respiratory compromise. The only form of definite management of hepatic hydrothorax is liver transplantation, and so all patients in which it is diagnosed warrant further evaluation for transplantation candidacy.
\end{abstract}

\section{Introduction}

Hepatic hydrothorax refers to a large accumulation of fluid in the pleural space (generally greater than $500 \mathrm{~mL}$ ), occurring in a patient with cirrhosis and evidence of portal hypertension. Exclusion of primary cardiopulmonary and malignant etiology of pleural effusion is generally required. The estimated prevalence of hepatic hydrothorax is $5-12 \%$ in patients with cirrhosis [1]. In terms of laterality, while classically thought of as an isolated right-sided effusion, which occurs in about $85 \%$ of cases, it can also occur as a left sided effusion (13\%) or as bilateral effusions (2\%) [2]. Furthermore, while its most common presentation is within patients with cirrhosis and severe ascites, there are reports of hepatic hydrothorax occurring in patients with only minimal ascites.

The general tolerance of cirrhotic patients to large volume ascites is often observed, owing to the inherent capacitance of the peritoneal cavity [3]. However, even small amounts of fluid in the pleural space can lead to respiratory symptoms, such as resting dyspnea, hypoxemia, pleuritic pain, and cough, due to the more restrictive nature of the thoracic cavity $[3,4]$.

\section{Pathogenesis}

The mechanism of hepatic hydrothorax is the subject of much debate. Several speculative mechanisms have been put forth, including (a) hypoalbuminemia resulting in decreased plasma oncotic pressure; (b) increased venous pressure in the azygous venous system; (c) transdiaphragmatic migration of peritoneal fluid via diaphragmatic lymphatic channels [3]. However, none of these mechanisms account for the right-sided predominance of hepatic hydrothorax. Moreover, the above stated processes occur commonly in cirrhotic patients, and would suggest that hepatic hydrothorax invariably be present in most cirrhotic patients. To the contrary, it remains a relatively uncommon complication of end stage liver disease.

The most widely accepted mechanism of hepatic hydrothorax is small subcentimeter diaphragmatic defects, present in the tendinous portions of the diaphragm. In the setting of increased intra-abdominal pressure (e.g. ascites, sneezing, coughing), small portions of peritoneum herniate through gaps in the muscle fibers of the diaphragm, and into the pleural space $[3,4]$. These herniations, called pleuro-peritoneal blebs, can rupture, and the ensuing defects serve as communication between the pleural and peritoneal cavity, through which fluid can directly traverse.

This mechanism has been corroborated by autopsy, video thorascopically [5], as well as with studies with peritoneal scintigraphy that demonstrate rapid migration of radiolabeled tracer from intraperitoneal injection into pleural fluid (tracer is not absorbed by the peritoneum, and thus the only mechanism of pleural accumulation is direct translocation through the anatomic defect) [6,7]. The direct passage of fluid between the two cavities accounts for the rapidity with which hepatic hydrothorax tends to re-accumulate following thoracentesis [6]. This mechanism furthermore provides explanation for the right-sided predominance of hepatic hydrothorax, given the increased likelihood of developmental defects in the right hemidiaphragm, the direct apposition of the liver to the right hemidiaphragm, allowing direct leakage of fluid through defects [1].

As studies of peritoneal scintigraphy have further suggested, the translocation of fluid is unidirectional. Negative intrathoracic pressure during the inspiratory phase of the respiratory cycle and increased intra-abdominal pressure favor the translocation of fluid from the peritoneal to the pleural space, and trapping of fluid into this space $[4,7]$. Hydrothorax occurs when the amount of fluid exceeds the reabsorptive capacity of the pleura $[2,4]$.

\section{Evaluation}

The evaluation of hepatic hydrothorax is centered on confirmation of pleural effusion and the exclusion of other etiologies that may result in a similar finding. The typical first step in this algorithm involves a chest radiograph, which can confirm the presence of pleural effusion. Although hepatic hydrothorax tends to involve the right hemithorax,

Correspondence to: Dr. Matthew D. Alvin, Department of Internal Medicine, Johns Hopkins Hospital, Baltimore, USA, E-mail: malvin2@jhmi.edu

Key words: hepatic hydrothorax, cirrhosis, liver transplantation

Received: May 29, 2016; Accepted: July 01, 2016; Published: July 04, 2016 
the presence of left-sided or bilateral effusion(s) does not exclude this diagnosis. Chest radiograph is followed by thoracentesis and other imaging modalities to rule out alternative causes of pleural effusion.

\section{Thoracentesis}

After obtaining pleural fluid, many diagnostic tests may be performed in order to further delineate the etiology of the pleural effusion. These tests include cell count and differential, gram stain, culture, protein, albumin, lactate dehydrogenase, glucose, bilirubin concentration, and $\mathrm{pH}$. In the case of hepatic hydrothorax, analyses may demonstrate a paucicellular transudate, normal glucose, and $\mathrm{pH}$ $>7.4$ [8] (Table 1). If other diagnoses are suspected, further diagnostic studies may be performed on the pleural fluid, including but not limited to, triglyceride level, amylase concentration, adenosine deaminase, and cytology $[2,4,8,9]$.

\section{Imaging modalities}

In the setting of suspected hepatic hydrothorax, computed tomography scans are primarily used to exclude lesions within the mediastinum, lung parenchyma, or pleura [2]. If malignancy is a concern and pleural fluid cytologic examination is non-diagnostic, thoracoscopy and pleural biopsy may be indicated [4]. In all patients with suspected hepatic hydrothorax, an echocardiogram should be completed to evaluate for cardiac causes of pleural effusion.

In cases where the diagnosis of hepatic hydrothorax is equivocal, scintigraphic studies using intraperitoneal injection of ${ }^{99 \mathrm{~m}} \mathrm{Tc}$-sulphur colloid or ${ }^{99 \mathrm{~m}} \mathrm{Tc}$-human serum albumin may be useful [10]. These radioisotopes travel in a unidirectional fashion, from the peritoneal cavity into the pleural space [11]. This study is optimally completed shortly after thoracentesis, when fluid tends to re-accumulate in the pleural cavity [12].

After the diagnosis of hepatic hydrothorax has been established, an abdominal ultrasound with Doppler study must be completed to assess the liver structure, visualize patency of the portal and hepatic veins, and identify ascites. If abdominal ascites is present, paracentesis should be performed [4].

\section{Management}

The mainstay of management for hepatic hydrothorax is similar to that for portal hypertensive ascites, and begins with sodium restriction and diuretic therapy.

\section{Sodium management}

Fluid accumulation in cirrhosis occurs as a consequence of several confounding processes, including portal hypertension resulting from distortion of hepatic architecture and neurohormonally mediated splanchnic arterial vasodilatation [13]. These processes lead to decreased effective circulating volume [14], leading to reflexive activation of systems that increase renal sodium avidity. Fluid retention is in large part mediated by this increase in renal sodium retention,

Table 1. Characteristics of pleural fluid in hepatic hydrothorax.

\begin{tabular}{|l|}
\hline Cell Count $<250$ polymorphonuclear cells $/ \mathrm{mm}^{3}$ \\
\hline Total Protein Concentration $<2.5 \mathrm{~g} / \mathrm{dL}$ \\
\hline Pleural fluid/serum total protein ratio $<0.5$ \\
\hline Pleural fluid/serum lactate dehydrogenase ratio $<0.6$ \\
\hline Serum-to-pleural fluid albumin gradient $>1.1 \mathrm{~g} / \mathrm{dL}$ \\
\hline Pleural fluid/serum bilirubin ratio $<0.6$ \\
\hline pH $7.40-7.55$ \\
\hline
\end{tabular}

which can be counteracted by maintaining negative sodium balance. This can be achieved either by decreasing sodium intake, or increasing urinary sodium excretion [14].

Patients are advised to restrict sodium intake to $88 \mathrm{mmol}$ per day (2000 mg) [15], which is usually well-tolerated [2]. The small portion of patients who achieve sufficient spontaneous naturesis can be managed with dietary sodium restriction alone [15], while the remainder require diuretic therapy [2]. Moreover, most patients opt in favor of some form of diuretic therapy to allow for liberalization of their sodium intake $[14,15]$. Diuretic therapy is usually initiated at furosemide 40 $\mathrm{mg}$ daily and spironolactone $100 \mathrm{mg}$ daily, and increased in a stepwise fashion, i.e., doubling the dose every 3-5 days, while maintaining the $100 \mathrm{mg} ; 40 \mathrm{mg}$ ratio until adequate clinical response is achieved [15]. Unresponsiveness to maximal diuretic therapy (furosemide $160 \mathrm{mg}$ daily, spironolactone $400 \mathrm{mg}$ daily) [16] is termed refractory hydrothorax, and is managed as described below. In addition, diuretic therapy may be limited by development of renal insufficiency, electrolyte derangements, or hepatic encephalopathy $[17,18]$.

\section{Thoracentesis}

Many large-volume effusions ( $>1.5 \mathrm{~L}$ ) may benefit from therapeutic thoracentesis. This should be completed with caution, and no more than $2 \mathrm{~L}$ of fluid should be removed, given the reported risk of re-expansion, pulmonary edema and hypotension $[12,19,20]$. Despite the significant and rapid relief of reported symptoms by patients, continued therapy with diuretics is recommended. In a select group of patients with sufficient natriuresis, this management alone is adequate. However, in patients with significant sodium retention (urine sodium $<10 \mathrm{mmol} /$ day), repeated thoracentesis every 2-3 weeks may be necessary for continued symptomatic relief [2]. In this patient population, repeated thoracenteses may impair quality of life and is associated with potential complications; consequently the placement of an indwelling pleural catheter (IPC) is often questioned.

While some advocate for the liberal use of IPCs, others suggest its use in cases when maximal medical therapy has failed, given the risk of IPC-related complications [21-24]. These complications include, but are not limited to, pleural infection, fluid loculation, drain site infections, acute renal failure, blockage, and mechanical failure of the drain itself [22]. In the case of hepatic hydrothorax, this palliative measure may be considered if Transjugular Intrahepatic Portosystemic Shunt (TIPS) is not possible, or as a bridge to liver transplantation [22].

\section{Transjugular intrahepatic portosystemic shunt (TIPS)}

Several studies have demonstrated the benefit of TIPS placement for management of refractory hepatic hydrothorax, mainly as a bridge to curative liver transplantation. In contrast to the more conservative measures detailed above, TIPS addresses the underlying cause of hepatic hydrothorax by creating a bypass channel between the portal system and the hepatic vein, in order to achieve portal decompression. Furthermore, the negative effects on systemic hemodynamics and renal function that limit the use of diuretic therapy and repeat large volume thoracentesis are not seen with TIPS [17]. To the contrary, TIPS leads to improvement in renal blood flow, urinary sodium and creatinine excretion [17]. Aggregates of small studies have demonstrated favorable clinical response in $60-70 \%$ of patients $[16,25]$. Small studies have demonstrated 1-year survival rate for refractory hydrothorax treated with TIPS was between $40-60 \%$ [26,27], and a 5 year survival of $15 \%$ [16] in another study. Prognostic factors for survival included age, pre-TIPS MELD score and creatinine clearance [15,26,27]. Surviving 
patients showed improvement in Child-Pugh score and renal function [26]. The most frequent complications of TIPS include portosystemic encephalopathy, due to direct passage of toxic metabolites normally cleared by the liver into systemic circulation [1], technical complications of the procedure, and shunt thrombosis or stenosis [21].

\section{Other options}

In patients who are non-responsive or intolerant of alternative therapies, chemical pleurodesis can be considered, which is often unsuccessful and results in recurrence of hydrothorax [21,28]. Other options include thorascopic repair of diaphragmatic defect [29]. Ultimately, these therapies are temporizing measures for management of symptoms resulting from hepatic hydrothorax, as the only form of definitive management is liver transplantation. For this reason, all patients with hepatic hydrothorax should undergo evaluation for liver transplantation candidacy.

\section{Conclusion}

Hepatic hydrothorax is a complication of end stage liver dysfunction, much in the same way as ascites, hepatic encephalopathy, or esophageal varices. In this cohort of patients, patients with hepatic hydrothorax have worse outcomes than would be predicted by MELD model predictions alone [30]. This highlights the importance of further study of this phenomenon. The diagnosis of hepatic hydrothorax in a patient should lead to warrant immediate evaluation for liver transplantation, as it is the only form of definitive management.

\section{Abbreviations}

Model for End Stage Liver Disease (MELD), Transjugular Intrahepatic Portosystemic Shunt (TIPS), Indwelling Pleural Catheter (IPC).

\section{References}

1. Campos S, Gomez D, Sofia C (2016) Transjugular Intrahepatic Portosystemic Shunt in Refractory... : European Journal of Gastroenterology \&Hepatology. LWW. European Journal of Gastroenterology and Hepatology.

2. Cardenas A, Kelleher T, Chopra S (2004) Review article: hepatic hydrothorax. Aliment PharmacolTher 20: 271-279. [Crossref]

3. Roussos A, Philippou N, Mantzaris GJ, Gourgouliannis KI (2007) Hepatic Hydrothorax: Pathophysiology Diagnosis and Management. J Gastroenterol Hepatol Journal of Gastroenterology and Hepatology 22: 1388-1393. [Crossref]

4. Lazaridis KN, Frank JW, Krowka MJ, Kamath PS (1999) Hepatic Hydrothorax: Pathogenesis, Diagnosis, and Management. The American Journal of Medicine 107: 262-267. [Crossref]

5. Huang P-M, Chang YL, Yang CY, Lee YC (2005) The Morphology of Diaphragmatic Defects in Hepatic Hydrothorax: Thoracoscopic Finding. The Journal of Thoracic and Cardiovascular Surgery 130: 141-145. [Crossref]

6. Serena A, Aliaga L, Richter JA, Calderon R, Sanchez L, Charvet MA (1985) Scintigraphic Demonstration of a Diaphragmatic Defect as the Cause of Massive Hydrothorax in Cirrhosis. Eur J Nucl Med European Journal of Nuclear Medicine 11: 46-48. [Crossref]

7. Ajmi S, Sfar R, Nouira M, Souguir A, Jmaa A, Golli L, et al. (2008) Role of the Peritoneopleural Pressure Gradient in the Genesis of Hepatic Hydrothorax. An Isotopic Study. Gastroenterol Clin Biol 32: 729-733. [Crossref]

8. Gurung P, Goldblatt M, Huggins JT, Doelken P, Nietert PJ (2011) Pleural Fluid Analysis and Radiographic, Sonographic, and Echocardiographic Characteristics of Hepatic Hydrothorax. Chest 140: 448-453. [Crossref]

9. Singh A, Bajwa A, Shujaat A (2013) Evidence-based review of the management of hepatic hydrothorax. Respiration 86: 155-173. [Crossref]

10. Benet A, Vidal F, Toda R, Siurana R, De Virgala CM, Richart C (1992) Diagnosis of Hepatic Hydrothorax in the Absence of Ascites by Intraperitoneal Injection of $99 \mathrm{~m}-\mathrm{Tc}$ -
Fluor Colloid. Postgraduate Medical Journal 68: 153. [Crossref]

11. Norvell JP, Spivey JR (2014) Hepatic hydrothorax. Clin Liver Dis 18: 439-449. [Crossref]

12. Cardenas A, Kelleher B, Chopra S (2016) Hepatic hydrothorax. Upto Date.

13. Walbaum B, Valda ML, Rada G (2016) Sodium restriction in patients with cirrhotic ascites: a protocol for a systematic review. Syst Rev 5: 78. [Crossref]

14. Morando F, Rosi S, Gola E, Nardi M, Piano S, Fasolato S, et al. (2015) Adherence to a Moderate Sodium Restriction Diet in Outpatients with Cirrhosis and Ascites: A Real Life Cross-sectional Study. Liver Int 35: 1508-1515. [Crossref]

15. Runyon BA, Practice Guidelines Committee, American Association for the Study of Liver Diseases (AASLD) (2004) Management of adult patients with ascites due to cirrhosis. Hepatology 39: 841-856. [Crossref]

16. Dhanasekaran R, West JK, Gonzales PC, Subramanian R, Parekh S, Spivey JR, et al (2010) Transjugular Intrahepatic Portosystemic Shunt for Symptomatic Refractory Hepatic Hydrothorax in Patients With Cirrhosis. Am J Gastroenterol 105: 635-641. [Crossref]

17. Rössle M, Gerbes AL (2010) TIPS for the treatment of refractory ascites, hepatorena syndrome and hepatic hydrothorax: a critical update. Gut 59: 988-1000. [Crossref]

18. Arroyo V, Ginès P, Gerbes AL, Dudley FJ, Gentilini P, Laffi G (1996) Definition and Diagnostic Criteria of Refractory Ascites and Hepatorenal Syndrome in Cirrhosis. International Ascites Club. Hepatology 23: 164-176. [Crossref]

19. Cantey EP, Walter JM, Corbridge T, Barsuk JH (2016) Complications of thoracentesis: incidence, risk factors, and strategies for prevention. Curr Opin Pulm Med 22: 378 385. [Crossref]

20. Ault MJ, Rosen BT, Scher J, Feinglass J, Barsuk JH (2015) Thoracentesis outcomes: 12-year experience. Thorax 70: 127-132. [Crossref]

21. Porcel JM (2014) Management of refractory hepatic hydrothorax. Curr Opin Pulm Med 20: 352-357. [Crossref]

22. Bhatnagar R, Reid ED, Corcoran JP, Bagenal JD, Pope S, et al. (2014) Indwelling Pleural Catheters for Non-malignant Effusions: A Multicentre Review of Practice. Thorax 69: 959-961. [Crossref]

23. Harris K, Chalhoub M (2012) The use of a PleurX catheter in the management of recurrent benign pleural effusion: a concise review. Heart Lung Circ 21: 661-665. [Crossref]

24. Mercky P, Sakr L, HeyriesL, Lagrange X, Sahel J, Dutau H. (2010) Use of a Tunnelled Pleural Catheter for the Management of Refractory Hepatic Hydrothorax: A New Therapeutic Option. Respiration 80: 348-352. [Crossref]

25. Spencer EB, Cohen DT, Darcy MD (2002) Safety and Efficacy of Transjugular Intrahepatic Portosystemic Shunt Creation for the Treatment of Hepatic Hydrothorax. Journal of Vascular and Interventional Radiology 13: 385-390. [Crossref]

26. Siegerstetter V, Deibert P, Ochs A, Olschewski M, Blum HE, Rössle M (2000) Transjugular Intrahepatic Portosystemic Shunt (TIPS) for Treatment of Hepatic Hydrothorax: Long-term Results in 40 Patients. Eur J Gastroenterol Hepatol. 13:529534. [Crossref]

27. Wilputte JY, Goffette P, Zech F, Godoy-Gepert A, Geubel A (2007) The outcome after transjugular intrahepatic portosystemic shunt (TIPS) for hepatic hydrothorax is closely related to liver dysfunction: a long-term study in 28 patients. Acta Gastroenterol Belg 70:6-10. [Crossref]

28. Elliot TB, Cárdenas A (2015) Contemporary Perspectives on the Pathophysiology and Management of Hepatic Hydrothorax.Curr Hepatology Rep 14: 212-218.

29. Luh SP, Chen CY (2009) Video-assisted Thoracoscopic Surgery (VATS) for the Treatment of Hepatic Hydrothorax: Report of Twelve Cases. J Zhejiang Univ Sci B 10: 547-551. [Crossref]

30. Badillo R, Rockey DC (2014) Hepatic hydrothorax: clinical features, management, and outcomes in 77 patients and review of the literature. Medicine (Baltimore) 93: 135-142. [Crossref]

Copyright: (C2016 Syed N. This is an open-access article distributed under the terms of the Creative Commons Attribution License, which permits unrestricted use, distribution, and reproduction in any medium, provided the original author and source are credited. 